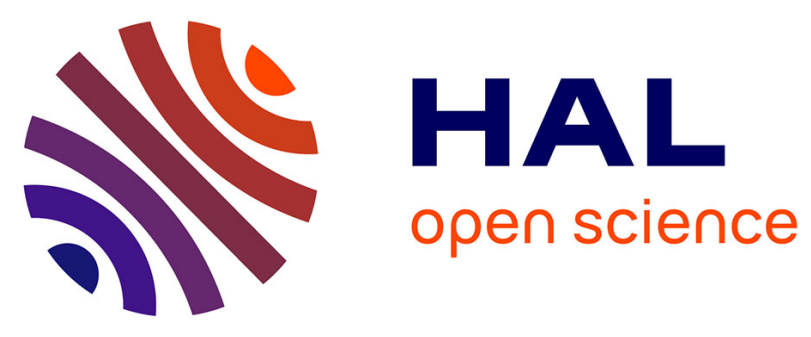

\title{
Convexifying Monotone Polygons
}

\author{
Therese C. Biedl, Erik D. Demaine, Sylvain Lazard, Steven M. Robbins, \\ Michael A. Soss
}

\section{To cite this version:}

Therese C. Biedl, Erik D. Demaine, Sylvain Lazard, Steven M. Robbins, Michael A. Soss. Convexifying Monotone Polygons. 10th Annual International Symposium on Algorithms \& Computation ISAAC'99, Kamakoti V (IMSC, India) Rangarajan K (MCC, India) Rama R (IIT, Madras, India) Boopal E (IIT, Madras, India), Dec 1999, Chennai, India. 10 p, 10.1007/3-540-46632-0_42 . inria00098832

\section{HAL Id: inria-00098832 https://hal.inria.fr/inria-00098832}

Submitted on 15 Dec 2009

HAL is a multi-disciplinary open access archive for the deposit and dissemination of scientific research documents, whether they are published or not. The documents may come from teaching and research institutions in France or abroad, or from public or private research centers.
L'archive ouverte pluridisciplinaire HAL, est destinée au dépôt et à la diffusion de documents scientifiques de niveau recherche, publiés ou non, émanant des établissements d'enseignement et de recherche français ou étrangers, des laboratoires publics ou privés. 


\title{
Convexifying Monotone Polygons
}

\author{
Therese C. Biedl ${ }^{1, \star}$, Erik D. Demaine ${ }^{1}$, Sylvain Lazard ${ }^{2, \star}$, \\ Steven M. Robbins ${ }^{3}$, and Michael A. Soss ${ }^{3}$ \\ 1 Department of Computer Science, University of Waterloo, Waterloo, Ontario N2L \\ 3G1, Canada, \{biedl, eddemaine\}@uwaterloo.ca \\ 2 INRIA Lorraine - LORIA, Projet ISA, 615 rue du jardin botanique B.P. 101, 54602 \\ Villers les Nancy Cedex, France, lazard@loria.fr \\ 3 School of Computer Science, McGill University, 3480 University Street, Montréal, \\ Québec H3A 2A7, Canada, \{stever, soss\}@cgm.cs.mcgill.ca
}

\begin{abstract}
This paper considers reconfigurations of polygons, where each polygon edge is a rigid link, no two of which can cross during the motion. We prove that one can reconfigure any monotone polygon into a convex polygon; a polygon is monotone if any vertical line intersects the interior at a (possibly empty) interval. Our algorithm computes in $O\left(n^{2}\right)$ time a sequence of $O\left(n^{2}\right)$ moves, each of which rotates just four joints at once.
\end{abstract}

\section{Introduction}

An interesting area in computational geometry is the reconfiguration of (planar) linkages: collections of line segments in the plane (called links) joined at their ends to form a particular graph. A reconfiguration is a continuous motion of the linkage, or equivalently a continuous motion of the joints, that preserves the length of each link. We further enforce that links do not cross, that is, do not intersect during the motion. For a survey of work on linkages where crossing is allowed, see the paper by Whitesides [9].

The case of noncrossing links has had a recent surge of interest. The most fundamental question [7] is still open: Can every chain be reconfigured into any other chain with the same sequence of link lengths? Here a chain is a linkage whose underlying graph is a path. Because reconfigurations are reversible, an equivalent formulation of the question is this: Can every chain be straightened, that is, reconfigured so that the angle between any two successive links is $\pi$ ? This question has been posed independently by several researchers, including Joseph Mitchell, and William Lenhart and Sue Whitesides [6]. It has several applications, including hydraulic tube and wire bending, and sheet metal folding [7].

At first glance, it seems intuitive that any chain can be "unraveled" into a straight line, but experimentation reveals that this is a nontrivial problem. Indeed, no such "general unraveling" motions have been formally specified. Because the problem is so elusive, it is natural to look at special classes of linkages and prove that at least they can be straightened. For example, consider the class of monotone chains, where every vertical line intersects the chain at a point or

* Research performed during a post-doctoral position at McGill University. 
not at all. Such a chain can easily be straightened by repeatedly (1) rotating the first link until it lines up with the second link, and (2) fusing these links together into a single "first link." This motion induces no crossings because it preserves monotonicity throughout.

This paper addresses the analogous question of straightenability for polygons (a linkage whose underlying graph is a cycle): Can every polygon be reconfigured into a convex polygon? In other words, can every polygon be convexified? This question was also raised by the researchers mentioned above. Note that a convex polygon can be reconfigured into any other convex polygon with the same clockwise sequence of link lengths, and hence the question is equivalent to the fundamental question for polygons [7]: Can every polygon be reconfigured into any other polygon with the same clockwise sequence of link lengths?

In this paper we focus on the case of monotone polygons. Similar to the case of chains, a polygon is monotone if the intersection of every vertical line with the interior of the polygon is an interval, that is, either a single vertical line segment, a point, or the empty set. See Fig. 1 for an example. A monotone polygon consists of two chains,

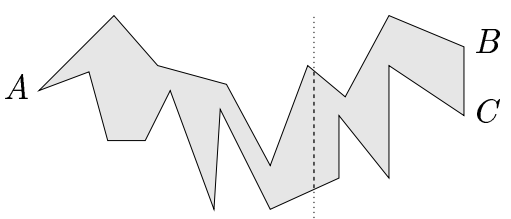

Fig. 1. A monotone polygon. the upper chain and the lower chain. Each chain is weakly monotone in the sense that the intersection with a vertical line is either empty, a single point, or a vertical edge. ${ }^{1}$ The left [right] ends of the upper and lower chains may be identical (like point $A$ in Fig. 1), or they may be connected by a vertical edge (like edge $(B, C)$ in Fig. 1). The vertical edge $(B, C)$ belongs to neither chain.

In contrast to monotone chains, it is nontrivial to convexify monotone polygons. In this paper, we show that this is possible by a fairly simple motion consisting of a sequence of $O\left(n^{2}\right)$ moves. We use just a single type of move, changing the angles of only four joints, which we show is the fewest possible. While the proof of correctness is nontrivial, our algorithm for computing the motion is simple and efficient, taking $O\left(n^{2}\right)$ time.

\subsection{Related Work}

Let us briefly survey the work on reconfiguring linkages whose links are not allowed to cross.

The most related result, by Bose, Lenhart, and Liotta [3], is that all monotoneseparable polygons can be convexified. A monotone-separable polygon is a monotone polygon whose upper and lower chains are separated by a line segment (connecting the common ends of the chains). Their motion involves translating almost all joints in the upper chain at once, and appears not to extend to general monotone polygons.

The only other result about convexifying classes of polygons is that every star-shaped polygon can be convexified [5]. A polygon is star-shaped if its bound-

\footnotetext{
${ }^{1}$ All straight (angle- $\pi$ ) vertices are removed, so there is no possibility of two adjacent vertical edges.
} 
ary is entirely visible from a single point. This motion rotates all joints simultaneously, and it seems difficult to find a motion involving few joints [10].

The remaining related results are for types of linkages other than polygons. For tree linkages, it is known that the answer to the fundamental problem is "no" [2]: There are some trees which cannot be reconfigured into other trees with the same link lengths and planar embedding. Indeed, there can be an exponential number of trees with the same link lengths and planar embedding that are pairwise unreachable. The complexity of determining whether a tree can be reconfigured into another remains open.

Another way to change the problem is to allow linkages in higher dimensions. If we start with a polygon in the plane, and allow motions in three dimensions, then every polygon can be convexified [8]. Indeed, a 1935 problem by Erdős asks whether a particular sequence of moves through 3D, each rotating only two joints (called a "flip"), converges in finite time. While the answer is positive, the number of moves is unfortunately unbounded in $n$. Recently, it was shown that $O(n)$ moves of a different kind suffice [1]; each rotates at most four joints.

If the polygon lies in three dimensions and we want to convexify it by motion through three dimensions, then it is surely not convexifiable if it is knotted. But there are unknotted polygons that cannot be convexified [1]. The complexity of determining whether a polygon in $3 \mathrm{D}$ can be convexified also remains open. Amazingly, Cocan and O'Rourke [4] have shown that every polygon in $d$ dimensions can be convexified through $d$ dimensions for any $d \geq 4$.

\subsection{Outline}

The rest of this paper is organized as follows. Section 2 begins with a more formal description of the problem. Section 3 describes our algorithm for computing the motion. Sections 4 and 5 prove its correctness and bound its performance, respectively. We conclude in Section 6.

\section{Definitions}

This section gives more formal definitions of the concepts considered in this paper: linkages, configurations, and motions.

Consider a graph, each edge labeled with a positive number. Such a graph may be thought of as a collection of distance constraints between pairs of points in a Euclidean space. A realization of such a graph maps each vertex to a point, also called a joint, and maps each edge to the closed line segment, called a link, connecting its incident joints. The link length must equal the label of the underlying graph edge. If a graph has one or more such realizations, we call it a linkage.

An embedding of a linkage in space is called a configuration of the linkage. In a simple configuration, any pair of links intersect only at a common endpoint, and in this case the links must be incident at this joint in the linkage. We consider only simple configurations in this paper. A motion of a linkage is a continuous movement of its joints respecting the link lengths such that the configuration of the linkage remains simple at all times. 
In this paper, we consider linkages embedded in the plane whose graph is a single cycle. The configurations are simple polygons, and so divide the plane into the exterior and interior regions (distinguished by the fact that the interior region is bounded). Joints of an $n$-link linkage are labeled $j_{0}, j_{1}, \ldots, j_{n-1}$ in a counterclockwise manner: traversing the boundary in sequence $j_{0}, j_{1}, \ldots, j_{n-1}$ keeps the interior region on the left. The joint angle $\theta_{i}$ is the interior angle at joint $j_{i}: \theta_{i}=\angle j_{i+1} j_{i} j_{i-1} \in(0,2 \pi)$. We call a joint straight if the joint angle is $\pi$, convex if the joint angle is strictly less than $\pi$, and reflex otherwise. A configuration is convex if none of its joints are reflex.

The question considered is whether every monotone configuration of a cyclic linkage (or polygon) can be convexified, that is, moved to a convex configuration. We show this is true by giving an algorithm to compute such a motion.

\section{Algorithm}

As input, the algorithm requires a description (that is, the joint coordinates) of a polygon with $n$ links. In each main step, the algorithm computes a sequence of $O(n)$ moves that ultimately straighten a joint. This joint angle is then held fixed so that it remains straight forever after, effectively reducing the number of joints. As the algorithm continues, the configuration has fewer and fewer nonstraight joints. We stop when no reflex joint is left, and so the polygon is convex as desired.

First we need some notation for basic geometric concepts. Let $p$ and $q$ be two points in the plane. Define $\|p q\|$ to be the Euclidean distance between $p$ and $q$. If $p$ and $q$ are distinct, define $\operatorname{ray}(p, q)$ to be the ray originating at $p$ and passing through point $q$. We use "left" and "right" in two different senses, one for points and one for rays. A point $r$ is left or right of $\operatorname{ray}(p, q)$ if it is strictly left or right (respectively) of the oriented line supporting $\operatorname{ray}(p, q)$. A point $p$ is left or right of point $q$ if $p$ has a strictly smaller or larger $x$ coordinate than $q$, respectively. In both cases, we use nonstrictly left/right to denote left/right or equality, i.e., neither left nor right.

The algorithm works as follows:

\section{Algorithm Convexify}

- Until the polygon is convex:

- Find a rightmost reflex joint (a joint with maximum $x$ coordinate, breaking ties arbitrarily).

- Relabel joints counterclockwise along the polygon so that this rightmost reflex joint is $j_{1}$, and all straight joints are ignored.

- If $j_{1}$ belongs to the lower chain:

1. Compute the largest index $p$ such that $j_{2}, \ldots, j_{p-1}$ are right of ray $\left(j_{0}, j_{1}\right)$.

2. Until a joint has straightened:

(a) Perform the following move (see Fig. 2) until either the joints $j_{0}$, $j_{1}$, and $j_{p-1}$ become collinear; or one of the joints $\left\{j_{0}, j_{1}, j_{p-1}, j_{p}\right\}$ straightens: 
i. Fix the positions of joints $j_{p}$, $j_{p+1}, \ldots, j_{n-1}$, and $j_{0}$.

ii. Fix all joint angles except those at $j_{0}, j_{1}, j_{p-1}$, and $j_{p}$.

iii. Rotate $j_{1}$ clockwise about $j_{0}$.

iv. Move joint $j_{p-1}$ as uniquely defined by maintaining the distances $\left\|j_{1} j_{p-1}\right\|$ and $\left\|j_{p-1} j_{p}\right\|$.

v. Move joint $j_{i}, 2 \leq i \leq p-2$, as uniquely defined by maintaining the distances $\left\|j_{1} j_{i}\right\|$ and $\left\|j_{i} j_{p-1}\right\|$.

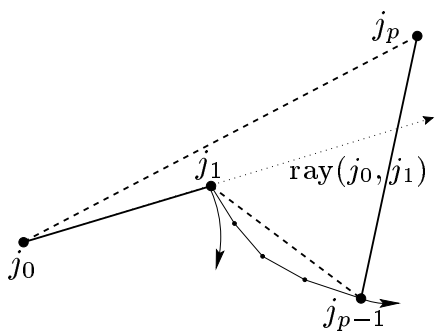

Fig. 2. The movement of joints $j_{1}$ and $j_{p-1}$. The thick dashed lines represent "virtual links" whose lengths are preserved.

(b) Update the coordinates of $j_{1}$ and $j_{p-1}$.

(c) If $j_{0}, j_{1}$, and $j_{p-1}$ are collinear, then decrement $p$, because with the new positions, $j_{p-1}$ is on $\operatorname{ray}\left(j_{0}, j_{1}\right)$. Also update the coordinates of the new $j_{p-1}$.

3. Update the coordinates of any remaining joints that have moved.

- If $j_{1}$ belongs to the upper chain, the algorithm is similar.

First let us justify that the algorithm is well-defined.

Lemma 1. The definition of $p$ in Step 1 is well-defined and at least 3.

Proof. Because $j_{1}$ is reflex, $\operatorname{ray}\left(j_{0}, j_{1}\right)$ must intersect the polygon elsewhere than the segment $\left(j_{0}, j_{1}\right)$. This implies that there are joints on both sides of the ray, and hence $p$ is well-defined. Furthermore, because $j_{1}$ is reflex and the joints are oriented counterclockwise on the polygon, $j_{2}$ is right of ray $\left(j_{0}, j_{1}\right)$. Hence, 2 is a valid value for $p-1$, so $p \geq 3$.

Note further that the number of simultaneously rotating joints (four) is the best possible, because any motion of a polygon that rotates just three joints reconfigures a virtual triangle, which is rigid.

\section{Proof of Correctness}

In this section, we prove the following theorem.

Theorem 1. Given any monotone polygon, Algorithm Convexify computes a convexifying motion, during which the polygon remains simple and monotone.

The difficulty is in showing that the polygon remains monotone and simple during the motion. For the remainder of this section, assume without loss of generality that the link $\left(j_{0}, j_{1}\right)$ is on the lower chain. First we need some trivial but important observations.

Lemma 2. During each move, no reflex angle becomes convex and no convex angle becomes reflex. 
Proof. Because all other joint angles are fixed, any such transition means that a joint $j_{0}, j_{1}, j_{p-1}$, or $j_{p}$ straightens, which stops the move.

Lemma 3. Joints $j_{2}, \ldots, j_{p-1}$ are convex.

Proof. Consider such a joint $j_{i}$. Because $j_{1}$ is a rightmost reflex vertex, $j_{i}$ is convex if it is right of $j_{1}$. By monotonicity and the property that $j_{i}$ is right of ray $\left(j_{0}, j_{1}\right), j_{i}$ cannot be left of $j_{1}$. The only case that remains is when $j_{i}$ has the same $x$ coordinate as $j_{1}$. Because we ignore straight joints, $j_{i}$ must in fact be $j_{2}$ in this case. Because $j_{1}$ is reflex, $j_{2}$ must be below $j_{1}$. Now $j_{3}$ must be strictly right of $j_{2}$, and hence the angle at $j_{2}$ is convex.

Next we need a general result about quadrangles.

Lemma 4. [1] Consider a simple quadrangle $j_{0}, j_{1}, j_{2}, j_{3}$ (in counterclockwise order) with $j_{1}$ reflex. (See Fig. 3.) Let $\theta_{i}$ denote the interior angle at joint $j_{i}$. If the linkage moves so that $j_{1}$ rotates clockwise about $j_{0}$, then $\theta_{1}$ decreases and $\theta_{i}$ increases for all $i \in\{0,2,3\}$, until $\theta_{1}$ straightens. In other words, all of the angles approach $\pi$.

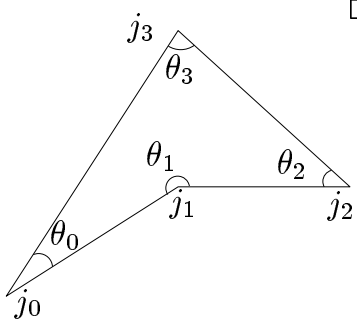

Fig. 3. Illustration of Lemma 4.

By the definition of $p, j_{1}$ is reflex in the quadrangle $Q=\left(j_{0}, j_{1}, j_{p-1}, j_{p}\right)$, so we can apply Lemma 4 to $Q$ and obtain the following result about our motion:

Lemma 5. During each move in Step 2a, $j_{p-1}$ rotates counterclockwise about $j_{p}$, and the joint angles $\theta_{1}$ and $\theta_{p-1}$ both approach $\pi$.

Next we analyze the movement of $j_{1}$ relative to $j_{p-1}$ 's reference frame, determined by fixing the position of $j_{p-1}$ and keeping the axes parallel to the world frame's. This can be visualized by imagining that during the motion we translate the entire linkage so that $j_{p-1}$ stays in its original position.

Lemma 6. $j_{1}$ rotates counterclockwise about $j_{p-1}$.

Proof. Consider the relative movement of $j_{1}$ and $j_{p}$ about $j_{p-1}$. Joint $j_{p}$ is rotating counterclockwise about $j_{p-1}$ and the angle $\angle j_{1} j_{p-1} j_{p}$ is increasing by Lemma 5 . Hence, $j_{1}$ must also be rotating counterclockwise about $j_{p-1}$.

We are now in the position to prove that the polygon remains simple and monotone throughout the motion.

Proof (Theorem 1). The only way that simplicity or monotonicity can be violated is that either a link intersects another link, or a vertical link rotates in the "wrong" direction. The wrong direction for link $\left(j_{i}, j_{i+1}\right)$ on the lower [upper] chain is when $j_{i+1}$ becomes left [right] of $j_{i}$. Consider the first time at which a link intersects another, or a vertical link rotates in the wrong direction.

Suppose first that a vertical link $\left(j_{i}, j_{i+1}\right)$ rotates in the wrong direction. Because only joints $j_{1}, \ldots, j_{p-1}$ move, we must have $0 \leq i \leq p-1$. We distinguish three cases: 
Case 1: Link $\left(j_{0}, j_{1}\right)$ is vertical

Because $j_{1}$ is reflex and $j_{2}$ is nonstrictly right of $j_{1}, j_{1}$ must be above $j_{0}$.

But $j_{1}$ rotates clockwise about $j_{0}$, so monotonicity is preserved.

Case 2: $\operatorname{Link}\left(j_{1}, j_{2}\right)$ is vertical

Because $j_{1}$ is reflex, $j_{1}$ is above $j_{2}$. By Lemma $6, j_{1}$ rotates counterclockwise about $j_{p-1}$. Hence, the rigid triangle $j_{p-1} j_{1} j_{2}$ rotates counterclockwise about $j_{p-1}$. Thus because the link $\left(j_{1}, j_{2}\right)$ is vertical, $j_{1}$ is above $j_{2}$, and $j_{p-1}$ is (nonstrictly) right of $j_{1}, j_{1}$ moves left of $j_{2}$. Thus, monotonicity is preserved.

Case 3: Link $\left(j_{i}, j_{i+1}\right)$ is vertical, $2 \leq i \leq p-1$

We show that joints $j_{i}$ and $j_{i+1}$ are both convex. First, if $i \leq p-2$, then this follows by Lemma 3 . Second, if $i=p-1$, then $j_{p-1}$ and $j_{p}$ must be right of $j_{1}$ in order for them to be on opposite sides of ray $\left(j_{0}, j_{1}\right)$. Hence, $j_{p-1}$ and $j_{p}$ must be convex because they are right of $j_{1}$ which is a rightmost reflex vertex.

Thus, joints $j_{i-1}$ and $j_{i+2}$ are both left of the link $\left(j_{i}, j_{i+1}\right)$; that is, $\left(j_{i-1}, j_{i}\right)$ belongs to the lower chain and $\left(j_{i+1}, j_{i+2}\right)$ belongs to the upper chain. This means that link $\left(j_{i}, j_{i+1}\right)$ joins the top chain to the bottom chain (like link $B C$ in Fig. 1). The polygon remains monotone no matter which way the link moves.

Now suppose that two links intersect each other, but the polygon remained simple and monotone before this time. By Lemma 5 , the joint angles $\theta_{1}$ and $\theta_{p-1}$ approach $\pi$, and hence the chain of moving links $\left(j_{0}, \ldots, j_{p}\right)$ cannot selfintersect. Hence, the only concern is whether any of these links could intersect the rest of the polygon.

In the following, refer to Fig. 4 . Let $u$ denote the original position of $\operatorname{ray}\left(j_{0}, j_{1}\right)$, and $v$ denote the downward vertical ray emanating from $j_{0}$. Let $W$ be the wedge right of ray $u$ and left of ray $v$.

Joint $j_{1}$ starts on the boundary of $W$; because it rotates clockwise about $j_{0}$, it enters region $W$ at the start of the move. By the choice of $p$, joints $j_{2}, \ldots, j_{p-1}$ all lie to the right of $u$. These joints must also lie to the left of $v$, because otherwise the polygon would not be monotone. Thus, after the move starts, the chain $j_{1}, \ldots, j_{p-1}$ lies inside $W$.

We now argue that the only joints that can be inside $W$ are $j_{1}, \ldots, j_{p-1}$. We know that $j_{0}$ and $j_{p}$ are not interior to $W$. If some other joint lies inside $W$, the chain must cross one of the boundaries of $W$. The chain cannot cross ray $v$, because that would violate monotonicity. Nor can the chain cross ray $u$, because that would require a reflex vertex to the right of $j_{1}$ or a violation of monotonicity. Because none of $j_{p}, \ldots, j_{n-1}, j_{0}$ are moving, this chain remains outside $W$ during the motion.

To establish that the polygon remains simple, we claim further that the joints $j_{1}, \ldots, j_{p-1}$ never leave $W$. Suppose to the contrary that one does. Let $j_{i}$ be the first such joint to leave $W$. It must reach either ray $u$ or ray $v$. Consider each possibility in turn.

$j_{i}$ crosses $v$ : Because $j_{1}$ stays reflex (unless it straightens which stops the move), it cannot be the first to cross $v$. If $j_{i}$ crosses $v$ for $1<i<p$, then we have both $j_{0}$ and $j_{i}$ left of $j_{1}$, so the chain is not monotone, a contradiction. 


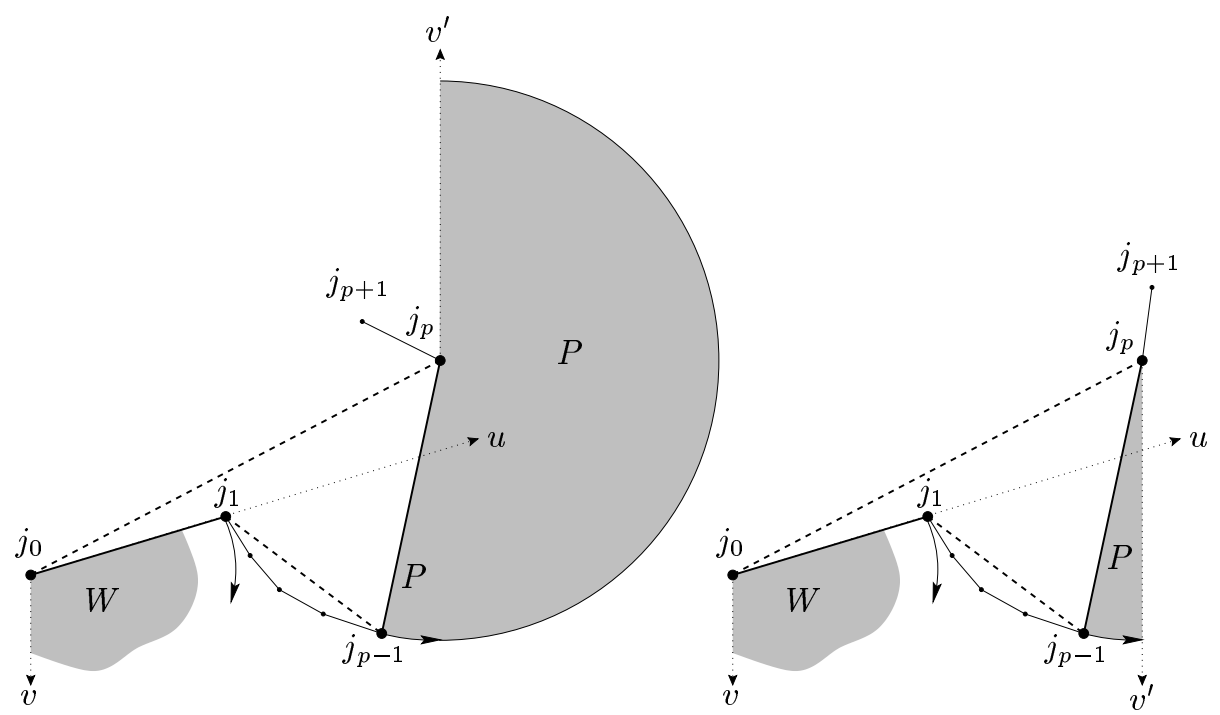

Fig. 4. Definition of $v, v^{\prime}, W$, and $P$. (Left) When $\left(j_{p}, j_{p+1}\right)$ is not on the lower chain. (Right) When $\left(j_{p}, j_{p+1}\right)$ is on the lower chain.

$j_{i}$ crosses $u$ : Because $j_{1}$ rotates clockwise about $j_{0}$, it never crosses $u$. If $j_{2}$ crosses, $j_{1}$ cannot be reflex, a contradiction. If $j_{p-1}$ crosses, the move must have already stopped from $j_{0}, j_{1}$, and $j_{p-1}$ becoming collinear because $j_{1}$ rotates clockwise about $j_{0}$. Finally, if $j_{i}$ crosses $u$ for $2<i<p-1, j_{i}$ must be reflex because $j_{i-1}$ and $j_{i+1}$ are both right of $u$, contradicting Lemma 3 .

Hence, the links of the chain $j_{0}, \ldots, j_{p-1}$ always remain inside $W$, so they cannot intersect links outside of $W$.

This leaves just one moving link, $\left(j_{p-1}, j_{p}\right)$. By Lemma $5, j_{p-1}$ rotates counterclockwise about $j_{p}$. Define $v^{\prime}$ to be the vertical ray emanating from $j_{p}$ that points away from the interior of the polygon, preferring upward if both directions are possible. Thus, $v^{\prime}$ points downward [upward] when the link $\left(j_{p}, j_{p+1}\right)$ is [not] on the lower chain.

Let $P$ be the pie wedge bounded by the link $\left(j_{p-1}, j_{p}\right)$, the ray $v^{\prime}$, and the counterclockwise circular arc, centered at $j_{p}$, starting at $j_{p-1}$ and ending on $v^{\prime}$ (at distance $\left\|j_{p} j_{p-1}\right\|$ from $j_{p}$ ). Because monotonicity is preserved up to this point, $P$ is empty of nonmoving links. $P$ also contains the entire sweep of $\left(j_{p-1}, j_{p}\right)$ : if $v^{\prime}$ points upward, $j_{p-1}$ cannot cross $v^{\prime}$ without first becoming collinear with $j_{0}$ and $j_{1}$; if $v^{\prime}$ points downward, it cannot cross without first straightening $j_{p}$.

\section{Time and Move Bounds}

Finally we establish the time and move bounds on the algorithm. Our model of computation is a real random-access machine supporting comparisons, basic arithmetic, and square roots.

Lemma 7. Each iteration of Step 2 takes constant time. 
Proof. The computations in this step are computing which of the five candidate events for stopping the motion occurs first (Step 2a), and updating $O(1)$ coordinate positions (Steps $2 \mathrm{~b}$ and 2c).

In order to compute the halting event, we define the following (refer to Fig. 5). Here $x^{*}$ denotes that $x$ changes during the move.

$$
\begin{aligned}
& a=\left\|j_{0} j_{1}\right\|, \quad b=\left\|j_{1} j_{p-1}\right\|, \quad c=\left\|j_{p-1} j_{p}\right\|, \\
& d=\left\|j_{p} j_{0}\right\|, \quad m^{*}=\left\|j_{1} j_{p}\right\|, \\
& \varphi_{0}^{*}=\angle j_{p} j_{0} j_{1}^{*}, \quad \varphi_{1}^{*}=\angle j_{0} j_{1} j_{p-1}^{*} \\
& \varphi_{p-1}^{*}=\angle j_{1}^{*} j_{p-1}^{*} j_{p}, \quad \varphi_{p}^{*}=\angle j_{p-1}^{*} j_{p} j_{0}
\end{aligned}
$$

Note that $m^{*}$ increases during the move, because $j_{1}^{*}$ rotates clockwise about $j_{0}$, increasing $\varphi_{0}^{*}$ and thus (by law of cosines) $m^{*}$. Hence, we parameterize the move by the diagonal distance $m^{*}$. More precisely, we de-

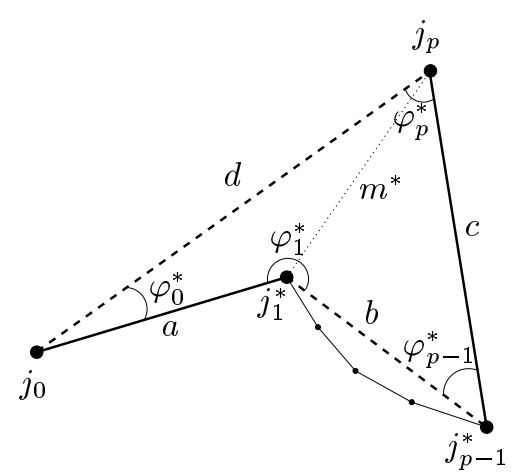

Fig. 5. Illustration of the proof of Lemma 7. termine the halting event by computing the value of $\left(m^{*}\right)^{2}$ for each of the five candidate events, and choosing the event with smallest $\left(m^{*}\right)^{2}$.

Now the event that $j_{i}$ straightens happens when its angle $\theta_{i}^{*}$ is $\pi$, and the event that $j_{0}, j_{1}^{*}$, and $j_{p-1}^{*}$ become collinear happens when $\varphi_{1}^{*}=\pi$. Note however that $j_{1}^{*}$ can only straighten after $j_{0}, j_{1}^{*}$, and $j_{p-1}^{*}$ become collinear (because $\angle j_{0}, j_{1}^{*}, j_{p-1}^{*}$ is initially reflex), and hence we do not need to consider this event. Using the law of cosines, we obtain the following solutions to these event equations:

$j_{0}$ straightens: $\left(m^{*}\right)^{2}=a^{2}+d^{2}+2 a d \cos \left(\angle j_{n-1} j_{0} j_{p}\right)$ and $\angle j_{n-1} j_{0} j_{p}<\pi$.

$j_{p-1}^{*}$ straightens: $\left(m^{*}\right)^{2}=b^{2}+c^{2}+2 b c \cos \left(\angle j_{p-2} j_{p-1} j_{1}\right)$.

$j_{p}$ straightens: Provided $\angle j_{0} j_{p} j_{p+1}<\pi$ (which is necessary for this event), $\left(m^{*}\right)^{2}$ is the solution of a quadratic polynomial involving $a, b, c, d$, and $\cos \left(\angle j_{0} j_{p} j_{p+1}\right)$. This reduces to an arithmetic expression involving square roots.

$j_{0}, j_{1}^{*}, j_{p-1}^{*}$ collinear: $\left(m^{*}\right)^{2}=\left(a c^{2}+b d^{2}-a b(a+b)\right) /(a+b)$, because $\cos \alpha_{1}^{*}=$ $-\cos \beta_{1}^{*}$.

The new joint coordinates can be computed as follows: Suppose that we know the new coordinates of joints $j_{i-1}$ and $j_{i}$ (initially $i=0$ ). Let $v$ be the vector from $j_{i}$ to $j_{i-1}$, rescaled to have the known length $\left\|j_{i} j_{i+1}\right\|$. Rotate this vector clockwise by the new $\theta_{i}$, which only involves cosines and sines of this angle, and then add it to the point $j_{i}$. The result is the new position of joint $j_{i+1}^{*}$. We can similarly compute the new coordinates of $j_{p-1}^{*}$ from the coordinates of $j_{p}$ and $j_{p+1}$. Thus, each update of $j_{1}^{*}, j_{p-1}^{*}$ (Step 2b), and possibly $j_{p-2}^{*}$ (Step 2c) takes constant time as desired.

Theorem 2. Algorithm Convexify computes $O\left(n^{2}\right)$ moves in $O\left(n^{2}\right)$ time.

Proof. By Lemma 7, any one move takes $O(1)$ time to compute. Any execution of Step 2 therefore takes $O(n)$ time, because initially $p \leq n-1$, and $p$ decreases 
with every move, until a joint straightens and Step 2 terminates. All other steps can also be implemented in $O(n)$ time. One iteration of the main loop thus takes $O(n)$ time. Because each iteration straightens a joint, the polygon becomes convex after $O(n)$ iterations. Hence, there are $O\left(n^{2}\right)$ moves, which are computed in $O\left(n^{2}\right)$ time.

\section{Conclusion}

We have presented an $O\left(n^{2}\right)$-time algorithm to compute a sequence of $O\left(n^{2}\right)$ moves, each rotating the minimum possible number of four joints at once, that reconfigures a given monotone polygon into a convex polygon with the same link lengths. By running the algorithm twice we can find a motion between any two monotone polygons with the same clockwise sequence of link lengths.

Several interesting open problems remain. Can our algorithm be improved to use $o\left(n^{2}\right)$ moves each rotating $o(n)$ joints? More generally, what is the tradeoff between the number of simultaneously rotated joints and the number of moves?

Our result adds to the class of polygons that are known to be convexifiable; previously, the only nontrivial classes were star-shaped polygons [5] and monotone-separable polygons [3]. A natural area of research is to explore more general classes of polygons. Is there a convexifiable class containing both monotone and star-shaped polygons (other than trivial classes like the union)?

Acknowledgments. We thank William Lenhart, Anna Lubiw, Godfried Toussaint, and Sue Whitesides for helpful discussions. This work was partially supported by FCAR and NSERC.

\section{References}

1. T. Biedl, E. Demaine, M. Demaine, S. Lazard, A. Lubiw, J. O'Rourke, M. Overmars, S. Robbins, I. Streinu, G. Toussaint, and S. Whitesides. Locked and unlocked polygonal chains in 3D. Manuscript in preparation, 1999. A preliminary version appeared in Proc. 10th ACM-SIAM Sympos. Discrete Algorithms, 1999, 866-867.

2. Therese Biedl, Erik Demaine, Martin Demaine, Sylvain Lazard, Anna Lubiw, Joseph O'Rourke, Steve Robbins, Ileana Streinu, Godfried Toussaint, and Sue Whitesides. On reconfiguring tree linkages: Trees can lock. In Proc. 10th Canadian Conf. Comput. Geom., Montréal, Aug. 1998.

3. Prosenjit Bose, William Lenhart, and Giuseppe Liotta. Personal comm., 1999.

4. Roxana Cocan and Joseph O'Rourke. Polygonal chains cannot lock in 4D. In Proc. 11th Canadian Conf. Comput. Geom., Vancouver, Aug. 1999.

5. H. Everett, S. Lazard, S. Robbins, H. Schröder, and S. Whitesides. Convexifying star-shaped polygons. In Proc. 10th Canadian Conf. Comput. Geom., Montréal, Aug. 1998.

6. W. J. Lenhart and S. H. Whitesides. Reconfiguring closed polygonal chains in Euclidean $d$-space. Discrete Comput. Geom., 13:123-140, 1995.

7. Joseph O'Rourke. Folding and unfolding in computational geometry. In Proc. Japan Conf. Discrete and Computational Geometry, Tokyo, Dec. 1998. To appear.

8. Godfried Toussaint. The Erdős-Nagy theorem and its ramifications. In Proc. 11th Canadian Conf. Comput. Geom., Vancouver, Aug. 1999.

9. Sue Whitesides. Algorithmic issues in the geometry of planar linkage movement. Australian Computer Journal, 24(2):42-50, May 1992.

10. Sue Whitesides. Personal communication, Oct. 1998. 